\title{
La construcción de competencias en ambientes virtuales de aprendizaje
}

\author{
Sandra Milena Morales Mantilla
}

\begin{abstract}
Resumen
La construcción de competencias es uno de los retos de la educación contemporánea; la sola definición del término ha dado origen a una cantidad importante de reflexiones, incluso las instituciones han generado modelos de operacionalización, sin embargo la realidad nos lleva a concluir que, hasta el momento, no podemos hablar de una pedagogía y una didáctica de las competencias. En este sentido, el texto se orienta desde cuatro preguntas clave: ¿Cómo construir competencias en ambientes virtuales de aprendizaje? ¿Cómo entender las competencias? ¿Cuál es el potencial de los ambientes virtuales para la construcción de competencias? ¿Cuáles serían las competencias a construir? Para contestar estos interrogantes, se realizó una revisión de bibliografía sobre el tema, con un completo análisis sobre el mismo, teniendo como resultado una propuesta de acción pedagógica y didáctica.
\end{abstract}

Palabras clave: educación a distancia, ambiente virtual de aprendizaje, competencias. 


\title{
Building skills in virtual learning environments
}

\begin{abstract}
Building skills is one of the challenges contemporary education faces. The term's mere definition has given rise to an important number of reflections, and even institutions have generated operational models. Nonetheless actual facts lead us to conclude that, so far, we can not talk about pedagogy and a didactic of skills. This text focuses on four key questions: How to build skills in virtual learning environments? How to understand skills? What is the potential of virtual environments for building skills? And which would be the skills to build? In order to answer to this, a bibliography review on the topic is made, and an analysis and a proposal of pedagogical didactic action are presented.
\end{abstract}

Key Words: Distance education, virtual learning environment and skills.

Recibido: 05 de julio 2011 Aceptado: 18 de noviembre 2011

\section{Introducción}

La construcción de competencias en la Educación a Distancia, concretamente, en ambientes virtuales de aprendizaje, además de una exigencia del sistema educativo colombiano se constituye en un reto y en una oportunidad innovadora. El presente texto indaga sobre las posibilidades de los entornos virtuales de aprendizaje para la construcción de competencias. Se estructura en dos momentos: conceptualización y definición de competencias a construir.

Las competencias como propósitos de la formación y ejes estructurantes de los currículos llegan a la educación por imposición, moda e incluso por contagio del mundo laboral (Perrenoud, 2003), en Colombia surgen como discurso del sistema de aseguramiento de la calidad a partir de una reunión no articulada de aportes de la lingüística y de otras disciplinas y, hoy en día, por un proceso de recontextualización emergen como procesos enmarcados en la actuación y la producción (Tobón 2006); a pesar de la génesis de las mismas, su discurso se ha posicionado con fuerza; 
sin embargo, es evidente que aún las prácticas pedagógicas y didácticas no reflejan total coherencia con estas.

La dinámica de cambio constante económico y social del mundo contemporáneo, exige a la educación un proceso de modernización centrado en las políticas enfocadas en la información y en los resultados (Gilomen, y otros; 2004: 9); dicho de otro modo, en el marco de la llamada sociedad del conocimiento, de los discursos de la educación de calidad, de la gestión de la calidad y sobre todo, de la evaluación con estándares internacionales, la Educación Superior se ve obligada a repensar su función formadora y a resignificar sus prácticas.

La Educación Superior a Distancia, de acuerdo con los planteamientos que inspiran el proceso de modernización educativa, debe experimentar una profunda renovación; más allá de fortalecer el paradigma educativo centrado en la actividad autónoma del estudiante, requiere innovar sus formas de mediación para el aprendizaje; esto es propiciar auténticos espacios para el aprendizaje autónomo, significativo y competente. Los ambientes virtuales de aprendizaje, hoy escenarios de la Educación a Distancia de punta, se constituyen en una oportunidad para la formación. Así mismo, el modelo e-learning, aprendizaje mediado en un entorno virtual (Barberá, 2008), adquiere especial pertinencia.

Educar en la sociedad del conocimiento y de la información implica enfrentar varios retos para garantizar su eficacia: el primero de ellos, asegurar el acceso a la información misma, dicho de otro modo, posibilitar a las personas la oportunidad de acceder a la Red, escenario por excelencia para la difusión de la información; por otra, comprender que el conocimiento y las informaciones se han convertido en la base de los procesos productivos... pero también se ha incrementado su importancia en diversos ámbitos del desempeño ciudadano (Tedesco, 2009: 71); en ese sentido, la educación de calidad para todos (cobertura con calidad), la educación a lo largo de la vida y la formación moral para la convivencia, parecen ser elementos decisorios para desenvolverse en la sociedad contemporánea e iniciar y permanecer en la vida laboral. Asuntos que llevan a concluir que la formación en la universidad actual debe apuntar a la formación de profesionales productivos y ciudadanos competentes.

\section{Metodología}

La presente reflexión se orienta desde la pregunta: ¿cómo construir competencias en ambientes virtuales de aprendizaje? Así mismo se formulan tres preguntas complementarias: ¿Cómo se entienden las competencias? 
¿Cuál es el potencial de los ambientes virtuales para la construcción de competencias? ¿Cuáles serían las competencias a construir? Para dar respuesta se realizó una concienzuda revisión bibliográfica a partir de la cual se establecen relaciones entre los ambientes virtuales de aprendizaje y la construcción de competencias.

Un ambiente virtual de aprendizaje se define como un espacio formativo ubicado en una plataforma tecnológica que facilita todas las interacciones necesarias para el aprendizaje en un entorno digital mediante TIC en la Red (Barberá, 2008); así mismo, es el lugar donde la clase virtual entendida como un grupo de alumnos matriculados en un curso de formación a distancia, mediante un medio tecnológico, interactúan entre sí, con su mediador, con un contenido y cuentan con herramientas comunicativas diversas y recursos académicos variados para conseguir el aprendizaje. Son tres los elementos claves del proceso de aprendizaje en la clase virtual: e-mediador, e-estudiante y el e-contenido; de la relación y la propuesta didáctica establecida entre estos tres elementos dependerá, en gran parte, el potencial del ambiente virtual para favorecer la construcción de competencias.

¿Cómo se entienden, entonces las competencias? Existen al menos tres concepciones aceptables de la noción competencias en educación. La primera las concibe como saber hacer. La segunda las entiende como capacidad y la tercera relaciona las dos concepciones anteriores.

La concepción de competencias, entendidas como saber hacer, surge de la relevancia otorgada al desempeño en el aprendizaje. En esta idea prima la eficacia en el desempeño, unida al acierto en la aplicación de los saberes.

En cuanto saber hacer, las competencias pueden expresarse como objetivos de la enseñanza, como conductas observables. Es decir, serían un tipo de objetivo que se expresa a través de un desempeño. Desde esta concepción, cualquier tipo de conocimiento que se manifieste en un desempeño específico se podría considerar una competencia; o por el contrario, cualquier saber hacer, al poder expresarse en una conducta observable, reflejaría una competencia.

Definir las competencias como saber hacer, representa una noción débil de las mismas, ya que las entiende como la suma de conocimientos de saber hacer o como la aplicación de conocimientos teóricos o prácticos. Le Boterf plantea que la eficacia de la formación basada en competencias radica en el cambio a una noción fuerte de las mismas. Las competencias, desde su visión, pueden entenderse como un saber hacer combinatorio, que se entiende a su vez como «la competencia» de una persona para construir «unas» competencias pertinentes (Le Boterf, 2001: 52). Es decir, el desempeño competente de una persona no está en directa relación con el saber hacer o la aplicación de conocimientos, sino se refiere, más bien, a su 
competencia para actuar de manera adecuada y eficaz frente a situaciones complejas y hasta cierto punto inéditas.

La segunda concepción entiende las competencias como capacidad, facultad genérica, potencialidad de todo ser humano. Esta concepción encuentra su raíz en la teoría del cambio propuesta por Aristóteles. El acto es una forma de ser distinta a la potencia, la perfección, la plenitud de ésta. Por su parte, la potencia puede expresarse de dos modos específicos: 1. Potencia natural para... 2. Hábito. Para convertirse en acto, la primera sufre una especie de alteración y la segunda ejerce algo que ya se poseía. En términos educativos, la potencia natural para... puede entenderse como la capacidad para el aprendizaje y el hábito como el saber aplicado. En el lenguaje de las competencias éstos no podrían ser considerados competencias, pues la primera (potencia) se considera un prerrequisito para la construcción de competencias y, el segundo (hábito) sería un estricto saber hacer; es decir, la competencia no es la potencia, como tampoco la aplicación de una potencia.

Desde esta perspectiva, «ninguna competencia se da desde un principio, las potencialidades del individuo sólo se transforman en competencias efectivas según los aprendizajes, que no se producen espontáneamente, por ejemplo, de acuerdo a una maduración del sistema nervioso, y no se realizan en el mismo grado en cada ser humano» (Perrenoud, 2003: 25).

La tercera concepción de las competencias relaciona las dos anteriores. Se refiere a la idea de las competencias entendidas desde el enfoque competenciadesempeño. La competencia puede entenderse como la potencia (capacidad) que puede transformarse en acto, a través de actuaciones (conductas observables, saber hacer) aunque, para ser precisos, éstas nunca serían manifestación de la totalidad de la competencia. Es decir, «el desempeño observado sería un indicador más o menos fiable de una competencia, que se supone más estable y que sólo se puede medir de manera indirecta» (Perrenoud, 2003: 24); dicho de otro modo, y siguiendo a Jonnaert (2005), la puesta en marcha de un conjunto diversificado y coordinado de recursos, tanto internos como externos, que elige, selecciona y moviliza la persona en un contexto determinado le permite dar un tratamiento exitoso a una situación específica, siendo en últimas, ésta puesta en marcha, la auténtica competencia.

Partiendo de las concepciones anteriores se propone un concepto de competencias alternativo. Las competencias pueden entenderse como la forma en que las personas movilizan los recursos que poseen para desempeñarse de manera exitosa frente a determinadas situaciones. En consecuencia, para la construcción de competencias resulta clave identificar los recursos que se requieren para su construcción; así como proponer diversas actividades y finalmente ofrecer las posibilidades de actuación que permitan la movilización de recursos. Para llegar a este concepto se identifican ocho características de las competencias: 
Característica 1. Las competencias son aprendizajes, dominios que las personas construyen gracias a los recursos que poseen, a las experiencias educativas, a las oportunidades de ejercitación y a las demandas de su contexto. Es decir, las competencias no son innatas, son aprendizajes adquiridos en el marco de un contexto disciplinar y de un entorno físico, político, histórico, cultural y lingüístico específico.

Característica 2. Las competencias son aprendizajes de carácter complejo. Son al menos cuatro las condiciones que definen el carácter complejo de las competencias: 1 . No constituyen una habilidad muy específica. 2. Requieren de la movilización de recursos para su adquisición y realización. 3. Suponen la definición de unas actividades que se deben realizar con competencia. 3. Exigen actuaciones competentes en el marco de unos estándares preestablecidos. 4. Superan los saberes para convertirse en recursos para formar nuevas competencias. Para comprender la primera de ellas, las competencias no constituyen una habilidad muy específica, un ejemplo: emitir un mensaje utilizando el correo electrónico a pesar de la dificultad aparente de dicho aprendizaje no constituye en sí mismo un dominio competencial en la medida en que es una habilidad específica, producto de un conocimiento y un procedimiento determinado. Podría constituirse en una competencia cuando su utilización es el resultado de una elección.

Las competencias como cualquier otro aprendizaje requieren para su adquisición de la existencia en el individuo de unos recursos (prerrequisitos): capacidades, conocimientos, rasgos de personalidad, actitudes, valores, etc., así como de los recursos que le ofrece el contexto: redes de información, relaciones, oportunidades, necesidades; pero a la vez requiere de nuevos recursos adquiridos en el proceso de aprendizaje; las competencias utilizan, integran y movilizan conocimientos (Perrenoud, 2003: 8) y otros recursos para su adquisición y actuación. Pero éstos no pueden ser considerados competencias. Los recursos cognitivos, las aptitudes intelectuales, las habilidades, los conocimientos de una persona

son indispensables para la adquisición de competencias, para la adaptación de las mismas a los distintos contextos y, especialmente, para enfrentar tareas complejas, exigentes y poco rutinarias.

Característica 3. Las competencias se manifiestan en desempeños y/actuaciones; en ese sentido requieren la definición de actividades o tareas contextualizadas que permitan su realización. Así mismo, la definición previa de estándares que permita la validación de la acción competente; dicho de otro modo, no basta con sentirse competente, la actuación competente debe ser verificada de acuerdo con estandarizaciones previamente establecidas. En palabras de Perrenoud, las competencias «sólo resultan pertinentes en situación, y cada situación es única, aunque se la pueda tratar por analogía con otras, ya 
conocidas» (Perrenoud, 2005: 11); es decir, las competencias se manifiestan en actuaciones y para ser tales exigen un desempeño competente en una situación y tiempo determinados. Para considerar un desempeño como competente éste debe manifestar al menos cuatro dominios:

1. Un saber, o lo que es igual una serie de conocimientos comprensivos que justifican y dan sentido a las acciones. 2. Un saber hacer, saber aplicado que responde a ciertos referentes de calidad compartidos socialmente. 3 . Un saber estar, que se refiere al campo actitudinal; es decir, a la evaluación que hace el sujeto sobre la situación. 4. Un saber actuar, que permite movilizar, combinar y transferir una serie de recursos en una situación compleja, en un contexto determinado y con vistas a una finalidad (Le Boterf, 2001: 118).

Característica 4. Las competencias en tanto objetivos de aprendizaje son dominios evaluables. En consecuencia para evaluar competencias se debe tener en cuenta: 1. Si las competencias son observables a través de los desempeños, pero los desempeños no reproducen totalmente la competencia, éstas deben evaluarse más allá del desempeño. Es decir, tener en cuenta además los conocimientos empleados, el acierto en la selección de procedimientos, los comportamientos asumidos, los valores puestos en acción, las actitudes, el control de emociones manifestado y el aprovechamiento de los recursos que ofrece el medio. 2. En la medida en que las competencias representan un vínculo entre los rasgos individuales y determinados comportamientos, es posible identificar algunos rasgos indicadores de las competencias (Bernal, 2003: 143) que funcionen como pistas que orienten la proximidad a la competencia. 3. Si las competencias son dominios que se aprenden y se expresan en contextos específicos, deben ser evaluadas en los mismos contextos en que se han aprendido y pueden expresarse, o por lo menos en marcos de simulación lo más parecidos posible a los contextos iniciales. 4 . Si las competencias son dominios que unas personas manejan mejor que otras y los hacen eficaces en situaciones determinadas y, a la vez, existen diversas formas de expresar la competencia, la evaluación de competencias debe hacerse con fines de mejoramiento personal, más que de comparación entre distintos sujetos. 5. Si la evaluación de competencias no puede ser la estrategia para clasificar aprendices y menos aún para medir niveles comparativos de dominio, resulta improbable poder igualar a las personas por ellas, ya que, en el dominio se sigue conservando en cada una un nivel, un estilo, un modo particular; en ese sentido, resulta pertinente identificar distintos niveles de gradación, esto es de dominio de la competencia, con el fin de que cada aprendiz pueda llegar al límite de sus posibilidades. 6. Conviene distinguir al menos tres momentos en la evaluación: a. La evaluación de los recursos para construir las competencias (conocimientos, saber hacer...), con el fin de identificar el nivel de instrucción en relación con el problema a solucionar. b. La evaluación de la construcción de las competencias, que permite acercarse a la competencia propiamente dicha 
al identificar el acierto en la selección de procedimientos, los comportamientos asumidos, los valores puestos en acción, las actitudes, el control de emociones manifestado y el aprovechamiento de los recursos que ofrece el medio; y, 3. La evaluación de los resultados, que permite valorar el desempeño en relación con los criterios de realización esperados.

Característica 5. Las competencias apuntan a una perspectiva didáctica de carácter transversal (Sarramona, 2004: 14) respecto a las tradicionales disciplinas académicas, lo que conlleva implicaciones a nivel curricular, didáctico y evaluativo. En primer lugar, implican una revisión de los propósitos de formación del currículo, su pertinencia, organización, etc. En segundo lugar, requieren un análisis de las metodologías empleadas con el fin de seleccionar aquellas que centren su atención en el estudiante y su proceso de aprendizaje. Y, por último, la evaluación pasa de ser una cuestión de las disciplinas para ser considerada una competencia de la institución.

A nivel práctico puede resultar interesante que al interior de cada disciplina se intente reflexionar en torno a la utilidad de la misma partiendo de situacionesproblema que pueda enfrentar el aprendiz en su vida y requiera de la movilización de los conocimientos que adquiere. A nivel educativo la formación de competencias resulta relevante en la medida que permiten enfrentar familias de situaciones.

Característica 6. Las competencias nos hacen competentes ypueden perfeccionarse. Bastien sostiene que: «un experto es competente, a la vez: -porque domina muy rápido y con seguridad las situaciones más corrientes, puesto que se dispone de esquemas complejos que pueden entrar inmediata y automáticamente en acción, sin vacilación, ni reflexión real; $y$, - porque es capaz, con un esfuerzo de reflexión razonable, de coordinar y diferenciar rápidamente sus esquemas de acción y sus conocimientos para enfrentar situaciones nuevas» (Bastien, 1997, 8).

Según este autor, se es competente cuando el desempeño alcanza el nivel de habilidad o hábito. En este sentido, «el límite entre la habilidad y la competencia puede ser en ocasiones indefinido» (Weinert, 2004, 123).

De la misma manera, se es competente cuando continúa estando presente la reflexión en el desempeño. "Las competencias son compatibles con una automatización total o parcial, pero no la exigen» (Perrenoud, 2003: 31); serán más eficaces en la medida que se asocien con una postura reflexiva (Perrenoud, 2003: 11). Le Boterf, por su parte, definirá como competente a aquella persona que ha pasado del saber hacer, al saber actuar.

Es decir, un sujeto competente sabe movilizar, combinar y transferir recursos individuales y de red, en una situación compleja y con vistas a una finalidad. De la misma manera, sabe construir a tiempo competencias pertinentes para gestionar situaciones cada vez más complejas (Le Boterf, 2001: 43). 
El sujeto competente es capaz de realizar un conjunto de actividades según criterios deseables; a la vez que sabe cómo prepararse para realizar una actividad teniendo en cuenta las condiciones y las modalidades de la misma.

Característica 7. Las competencias se definen. Se ha puntualizado, que cada contexto demanda determinadas competencias. Sin embargo, la relación que sostiene el individuo con el medio lo modifica y determina nuevas competencias.

De tal manera que resulta imposible definir todas las competencias que requiere un individuo para enfrentar con éxito las distintas situaciones de su vida. El reto es, por tanto, desarrollar al interior de cada disciplina disposiciones en los estudiantes para enfrentar situaciones en las que deban movilizar los recursos adquiridos.

\section{Los Ambientes virtuales de aprendizaje y la construcción de competencias}

¿Cuál es el potencial que ofrecen los ambientes virtuales para la construcción de competencias? Para responder a esta pregunta se analizará el potencial que ofrecen los ambientes virtuales de aprendizaje en relación a los tres elementos esenciales para la construcción de competencias: adquisición de recursos, propuesta de actividades y definición de actuaciones que permitan la movilización de recursos.

En cuanto a la capacidad de los ambientes de aprendizaje para favorecer en el aprendiz la adquisición de los recursos para la construcción de competencias se destaca su enorme posibilidad para acceder a la información. Su presencia en la red permite de manera permanente, rápida y económica acceder a la información; así mismo los multiformatos en que ésta se presenta: discursos oral y escrito, animación gráfica, video, simulaciones, conferencias múltiples, diálogos con expertos, etc., permiten la adquisición de los recursos respetando los distintos estilos cognitivos.

Así mismo, los ambientes virtuales de aprendizaje ofrecen la posibilidad de comunicación multimedial sincrónica y asincrónica con múltiples espacios comunicativos que permite la interactividad permanente y temática entre los agentes y la creación de redes de aprendizaje. En esta lógica relacional los recursos para la construcción de competencias se adquieren a la vez que se desarrollan habilidades generales como la indagación, la creación, la deliberación, el juicio crítico, el consenso, entre otras.

Con respecto a las actividades que propone el e-mediador para favorecer la construcción de competencias en el e-estudiante, el ambiente virtual de aprendizaje favorece el papel activo del aprendiz que participa en una comunidad 
abierta intercambiando de manera permanente preguntas, ideas y respuestas. Así mismo, su actividad puede realizarse en multicontextos: la red misma, su contexto de actuación, su actividad profesional, entre otras.

Finalmente la amplitud del marco geográfico presente en los ambientes virtuales de aprendizaje, permiten la actuación en lo global y en lo glocal; dicho de otro modo, el e- estudiante tiene la posibilidad de comprender de manera global las diversas situaciones que se le presentan (en razón a la cantidad de información que tiene a la mano, así como por la interacción que mantiene con personas de otras culturas y modos de vida) y, simultáneamente, actuar de manera glocal. Así mismo, el ambiente virtual permite que el emediador proponga actuaciones en ambientes reales (contextos de actuación del aprendiz) así como en ambientes simulados, lo que implica una actitud ecológica frente al tiempo invertido en la construcción de una competencia y en los posibles errores con consecuencias para las comunidades que se pueden presentarse en el aprendizaje.

\section{Competencias a construir: una propuesta a partir del potencial de los ambientes virtuales de aprendizaje}

¿Cuáles son las competencias a construir en ambientes virtuales de aprendizaje?

Se proponen tres tipos de competencias: competencias comunicativas, como respuesta a las demandas actuales del ICFES en Colombia; competencias transversales; entendidas éstas como aquellas competencias que deben construirse en la modalidad de educación a distancia en entornos virtuales y, en tercer lugar las competencias especificas, definidas por cada disciplina en el marco de sus propósitos y sus núcleos problémicos (en este trabajo se abordarán los dos primeros grupos de competencias).

Competencias comunicativas: El ICFES propone tres competencias básicas para la formación en Colombia: Interpretación, Proposición y Argumentación. En algún momento se llegó a pensar incluso, en estas competencias como «las competencias» exclusivas de la propuesta educativa del país; por fortuna se ha salido de esta confusión.

Las competencias comunicativas en entornos virtuales adquieren un sentido e importancia especial; las interacciones comunicativas que se establecen en este tipo de entorno de aprendizaje y que determinan en gran parte el éxito formativo en la modalidad, invitan a hacer una contextualización de las mismas.

Las Tecnologías de la Información y la Comunicación - TIC- , presentes en los ambientes virtuales de aprendizaje aportan al proceso formativo 
elementos transformadores a nivel comunicativo; el flujo permanente y en diferentes niveles de la comunicación, exigen al aprendiz virtual un dominio competencial comunicativo específico. Barberá, define cuatro flujos comunicativos en cuanto a la dirección de la comunicación en función del emisor y el receptor que evidencian el potencial comunicativo de los entornos virtuales de aprendizaje, así como su potencial para favorecer la construcción de competencias comunicativas; a saber: 1. Flujo de conexión: responde a la relación comunicativa 1x1 (aprendiz-aprendiz; aprendizcontenido; aprendiz-mediador). 2. Flujo de distribución: responde a la relación 1xN (uno a muchos; aprendiz-compañeros; mediador-aprendices; contenido-aprendices). 3. Flujo convergente: responde a la relación $\mathrm{Nx} 1$ (muchos a uno; aprendices-contenido; aprendices-mediador). 4. Flujo colectivo: responde a la relación $\mathrm{NxN}$ (muchos a muchos) y refiere a la construcción de comunidades a través del establecimiento de redes (Barberá, 2008: 49-50).

Dado el alto nivel comunicativo escritural y lector que se establece en los ambientes virtuales y los diversos flujos comunicativos allí presentes, la interpretación de textos, códigos e intenciones comunicativas resulta fundamental; así mismo, la necesidad de concretar ideas, construir hipótesis y debatir, exige la proposición y, finalmente, el principio de construcción colaborativa del conocimiento presente en los entornos virtuales de aprendizaje requiere el consenso y la deliberación; dicho de otro modo, la capacidad para argumentar las propias ideas y contraargumentar otras.

Competencias clave: El concepto competencia clave es utilizado por primera vez en el marco del proyecto DeSeCo (Definition and Selection de Competencies: Theoretical and Conceptual Foundations) realizado bajo los auspicios de la OCDE, cuyo propósito es definir un conjunto limitado de competencias fundamentales, para el desarrollo humano y el ejercicio político y económico de la autoridad, que sean confiables y comparables a nivel internacional y que ostenten fundamentos teóricos. En lo que respecta al propósito de este trabajo, se formulan tres competencias clave; es decir competencias fundamentales para la formación y el desempeño en ambientes virtuales de aprendizaje que permiten comparatividad internacional; son ellas:

Competencia digital: Entendida como el uso seguro y crítico de las Tecnologías de la Información y la comunicación para la formación, el trabajo, el ocio y la comunicación. Se formulan dos competencias específicas: competencia informacional (referida a la capacidad de interactuar con la información) y la competencia tecnológica (entendida como capacidad para usar los artefactos). El fin último de la competencia digital se establece en relación a los diferentes niveles de interacción con la información que se establecen en ambientes virtuales de aprendizaje: 1. Búsqueda, obtención y comprensión de la información (nivel informativo); 2. Producción personal y difusión 
pública del conocimiento (nivel creativo); 3. Comunicación e interacción social (nivel transformador).

Competencia ciudadana: Las nuevas relaciones con el medio y con los otros, que se establecen al hacer uso de las tecnologías de la información y la comunicación exigen un replanteamiento del ejercicio de la ciudadanía. Los ambientes virtuales de aprendizaje permiten vivir simultáneamente en dos realidades: el mundo como lugar propiamente dicho y la realidad online; es decir, el concepto de ciudadano del mundo planteado por Adela Cortina, en el contexto de los entornos virtuales se amplía, además de ciudadanos del mundo, somos ciudadanos en una realidad virtual en donde también somos sujetos de deberes y derechos; dicho de otro modo, como miembros de una comunidad de aprendizaje: multicultural y multiubicua, el aprendiz adquiere un sentido de pertenencia que supera las barreras culturales y espaciales, para identificarse con unos propósitos comunes en igualdad de derechos, deberes y oportunidades. Esta relación permite generar compromisos con lo global (conozco lo otro y genero solidaridad por su causa), pero a la vez, acción local (apropio y actúo en el propio contexto). En este orden de ideas, la interculturalidad como modelo relacional en la comunidad de aprendizaje imprime un nuevo carácter al ejercicio de la ciudadanía.

Competencia para la autogestión del conocimiento. Los entornos virtuales de aprendizaje centrados en la concepción de aprendizaje autónomo (e-estudiante constructor de su propio conocimiento, autor de su propia formación); comprometidos con la construcción de currículos integrados que promueven la investigación como práctica para la producción y estrategia para la construcción de conocimiento; conocedores del dinamismo en la circulación de la información y la evolución permanente del conocimiento y de las demandas del sector productivo, se constituyen en un escenario para favorecer la educación a lo largo de toda la vida.

\section{Conclusiones}

La construcción de competencias se constituye en un propósito de la Educación Superior, formar profesionales productivos y ciudadanos competentes es una demanda y exigencia de la educación de nuestro tiempo. Los entornos virtuales de aprendizaje entendidos como espacios formativos ubicados en una plataforma tecnológica que facilitan todas las interacciones necesarias para el aprendizaje en un entorno digital mediante las Tecnologías de la Información y la Comunicación - TIC- en la Red ofrecen un importante potencial para la construcción y actualización de competencias. La accesibilidad y dinamismo para el manejo de la información, así como la 
oportunidad de adecuación a los tiempos, espacios y necesidades formativas de las personas y las posibilidades que ofrece para simular diversos contextos de actuación, los convierten en escenarios por excelencia para la construcción y/o perfeccionamiento de competencias.

Las competencias, por su parte, se entienden como la forma en que la persona moviliza los recursos que posee para actuar de manera eficaz frente a distintas situaciones, lo que implica el dominio de recursos cognitivos, procedimentales, actitudinales, así como de los recursos que ofrece el contexto y la capacidad para movilizarlos de manera acertada en las situaciones que deba enfrentar.

Se formulan tres grupos de competencias para fortalecer en los ambientes virtuales de aprendizaje, a saber: competencias comunicativas: interpretación, proposición y argumentación, formuladas en función de las diversas interacciones comunicativas que se establecen en este tipo de entornos de aprendizaje; competencias clave: entendidas como aquellas competencias requeridas para desempeñarse en ambientes virtuales y a la vez construidas en este tipo de ambientes: digitales, ciudadanas y de autogestión del conocimiento; y las competencias disciplinares, definidas al interior de cada disciplina.

En este sentido, los ambientes virtuales de aprendizaje requieren innovar en sus formas de mediación para favorecer la construcción de competencias; en primer lugar, se propone identificar los recursos que se requieren para construir competencias e incluirlos en los currículos existentes; así mismo, proponer situaciones de aprendizaje que favorezcan el dominio competencial; definir unos estándares que permitan la validación de la acción competente y, finalmente establecer una propuesta de evaluación coherente, basada en la resolución de tareas. La innovación pasa por la capacidad para diseñar contenidos y guías didácticas que, en el marco del ambiente virtual, se propongan favorecer la construcción de competencias.

En este escenario cobra especial importancia el papel del e-mediador; es decir, el tutor encargado de diseñar la propuesta de aprendizaje con las condiciones y características propias de la modalidad educativa; el e-estudiante, quien como centro del proceso formativo establece una relación con el e- contenido y su grupo de trabajo para construir de manera autónoma, significativa, contextualizada y crítica el conocimiento; y, finalmente el e-contenido que visibiliza el potencial de aprendizaje de los entornos virtuales por su carácter atractivo, dinámico e interactivo.

Estos tres actores: e-mediador. e-estudiante, e-contenido al estar ubicados en una plataforma virtual dinamizan la construcción de comunidades de aprendizaje y de innovación, generando sistemas de creación colectiva del conocimiento y propiciando la construcción de espacios de comunicación, 


\section{Sandra Milena Morales Mantilla}

La construcción de competencias en ambientes virtuales de aprendizaje, artículo de reflexión

diálogo y relación entre los actores, así como la organización y utilización de redes sociales de ámbito local y global, como estrategias de aprendizaje.

\section{Referencias bibliográficas}

Barberá, E. 2008. Aprender e-learning. Barcelona: Paidós.

Barnett, R. 2008. Para una transformación de la Universidad. Barcelona: Octaedro.

Bautista, J. 2000. La universidad en la encrucijada de la formación. Huelva: Hergué Editorial.

Bernal, A. 2003. "La construcción de la identidad personal como proyecto de educación moral. Supuestos teóricos y delimitación de competencias». En Teoría de la Educación. 15: 129-160.

Cubells, F. 1981. El concepto de acto energético en Aristóteles. Valencia: Facultad de Teología San Vicente Ferrer.

De Miguel, M. 2006. Metodologías de enseñanza y aprendizaje para el desarrollo de competencias. Madrid: Alianza Editorial.

Jonnaert, Ph., j. Barrete, S. Boufrahi, y D. Masciotra. 2005. "Contribution critique au devéloppement des programmes d'études: competences, constructivisme er interdisciplinarité». En, Revue des Sciences de l'éducation, volumen XXX(3): 667-696.

Leal, J., et al. 2009. Proyecto académico pedagógico solidario. Bogotá: UNAD.

Le Boterf, G. 2001. Ingeniería de las competencias, Barcelona: Gestión 2000

Morales, S. 2007. La educación de competencias para la convivencia en una sociedad plural. Valencia: Tesis doctoral.

Peiró, K. 2000. «El teletrabajo, una nueva forma de trabajar que se está implantando con fuerza en el entorno empresarial, industrial y rural». En, El teletrabajo. Un reto laboral en la sociedad de la información. pp. 27-49. Madrid: I.M.,F.S.E.

Perrenoud, P. 2003. Construir competencias desde la escuela. Santiago de Chile: J.C. Sáez. Editor.

Rychen, D. y L. Hersh. 2004. Definir y seleccionar las competencias fundamentales para la vida. México: Fondo de Cultura Económica. 


\section{Revista de}

\section{investigaciones UNAD}

Volumen 10. Número 2. Diciembre 2011

Sarramona, J. 2004. Las competencias básicas en la Educación obligatoria. Barcelona: CEAC.

Tedesco. J. 2009. Educar en la sociedad del conocimiento. Buenos Aires: Fondo de Cultura Económica.

Tobón, S. 2006. Competencias en la Educación Superior. Políticas hacia la calidad. Bogotá: ECOE Ediciones. 